\title{
Criterion bias and search sequence bias in word recognition
}

\author{
R. E. O'CONNOR and K. I. FORSTER \\ Monash University, Clayton, Victoria 3168, Australia
}

\begin{abstract}
Six experiments that were designed to test the adequacy of criterion bias explanations of the word frequency effect and the semantic priming effect are reported. It was found that criterion bias models correctly predicted higher error rates in a lexical decision task for nonwords that were misspelled versions of high-frequency words (e.g., MOHTER), rather than low-frequency words (e.g., BOHTER). Also correct was the prediction of increased error rates for misspelled words preceded by a semantically related word (e.g., NURSE-DOTCOR). However, in a misspelling decision task (in which the subject must decide whether the stimulus is a word, a misspelled word, or a nonword), it can be argued that criterion bias should be inoperative, since correct responses must be delayed until all orthographic information has been checked; this should eliminate both frequency and semantic priming effects. This was found not to be the case; clear frequency and priming effects were obtained for both words and misspelled words.
\end{abstract}

The notion of bias plays a key role in current attempts to explain many of the major characteristics of the word recognition process. The essence of this notion is that the perceptual system is biased in some way so that cer. tain percepts are more easily formed than others. One form of bias is referred to as criterion bias and was first proposed by Broadbent (1967) to explain how common words were identified more readily than were lowfrequency words. He suggested that subjects were "biased in such a way as to accept a smaller amount of evidence before deciding in favour of a probable word" (Broadbent, 1967, p. 3). Morton's $(1969,1970)$ logogen theory incorporates such a mechanism to account for the effects of both linguistic context and word frequency.

A quite different form of bias has been proposed within the context of ordered-search models of lexical access. In these models, recognition involves comparing a representation of the stimulus with a series of lexical entries in a mental dictionary (Forster, 1976; Rubenstein, Garfield, \& Millikan, 1970; Stanners \& Forbach, 1973). Bias is introduced into this system if the search through the set of lexical entries deviates in any way from a purely random search. Thus if there is a bias to search through the entries for high-frequency words first, the frequency effect can be explained. Or, if there is a bias to search through the entries for words that are semantically related to a context word, then the effects of semantic priming can be explained (Forster, 1976). This second kind of bias we will refer to as search sequence bias. The purpose of the present paper is to explore the differences between these two theoretical devices in terms of their capacity to explain the effects of frequency and semantic relatedness on both latencies and error rates in word classification tasks.

In Morton's model (Morton, 1970), word recognition is accomplished by a system of logogens, or word detec- tors, each of which is capable of signaling the presence of a particular word. These logogens act like counting devices; the value of each logogen is incremented whenever attributes of their associated words are input to the logogen system. For example, the word "cat" might first have the orthographic feature "three-letter word" extracted; this feature would then be input to the logogen system, which would cause an increase in the value of all those logogens that correspond to three-letter words. Then the "initial-C" attribute might be extracted, and the logogens of all words beginning with $\mathrm{C}$ would be incremented.

This process continues until the count in one logogen is incremented in excess of its particular critical or threshold value, at which point the corresponding word is recognized and at the same time all other logogens are inhibited from exceeding their thresholds.

The effect of word frequency (i.e., that commonly occurring words are identified more rapidly than are infrequently occurring words) is accounted for by the proposal that the logogen thresholds are determined by the frequency of their corresponding word. The logogens of high-frequency words have relatively low thresholds and thus require fewer sensory attributes to be processed before exceeding threshold. Low-frequency words, on the other hand, may require almost all of their sensory attributes to be input to the logogen system before their logogens fire.

Meyer and Schvaneveldt (1976) have used this mechanism to explain the semantic priming effect, in which a target word such as "cat" is recognized faster if it is preceded by a semantically related word such as "dog". Meyer and Schvaneveldt suggested that logogen discharge causes temporary increases in the activation levels of the logogens of semantically related words. Thus when the related word is subsequently presented, 
fewer attributes are required to be input to the logogen system before the appropriate logogen exceeds threshold and the word is recognized.

It should be noted that the two effects, frequency and semantic priming, are explained by the same basic mechanism, with high-frequency words being recognized faster than low-frequency words because the logogens of high-frequency words have lower criteria for discharge, and semantically related words being recognized faster than unrelated words because semantically related words have boosted activation levels. This particular aspect of the theory has recently been subjected to test by Becker and Killion (1977). Using a lexical decision task, Becker and Killion demonstrated an interaction between stimulus quality and semantic priming, with low stimulus intensity magnifying the priming effect. Meyer, Schvaneveldt, and Ruddy (1975) reported similar results when using a superimposed dot pattern to impair stimulus quality. The logogen model can account for this result by proposing that low stimulus quality serves to reduce the rate at which feature extraction occurs. As semantically primed logogens require fewer features for discharge than do nonprimed logogens, any decrease in the rate of feature input will be expressed as an increase in the difference in word recognition latency between primed and nonprimed words. However, when the variable of word frequency was substituted for that of semantic relatedness, Becker and Killion found that the manipulation of stimulus quality no longer appeared to produce interactive effects (see also Stanners, Jastrzembski, \& Westbrook, 1975). Because frequency is also assumed to affect identification time by way of a difference in the number of features that are required to initiate logogen discharge (high-frequency logogens having lower thresholds of activation than low-frequency logogens), an interaction should also have been found between word frequency and stimulus quality.

A further difficulty for logogen theory has been provided by the work of Becker (1979) and Bednall (1978; for discussion, see Forster, 1976). They have independently reported finding an interaction between semantic priming and word frequency. A straightforward interpretation of the logogen model (e.g., Becker, 1979) predicts that the effect of priming a logogen should combine in a simple way with the preexisting bias corresponding to word frequency, so that the effects of frequency and priming would be additive, not multiplicative. However, the model could be modified to account for this result, for example, by stipulating that the amount of activation produced by priming is proportional to the preexisting difference between the activation resting level and dis. charge threshold.

Although these experimental results do not lead to a rejection of the logogen model, they do emphasize that there are problems in trying to explain both frequency and priming effects in terms of criterion bias, that is, a reduction in the number of features that need to be extracted. A closer examination of the role of criterion bias therefore seems desirable, and the experiments reported in this paper were conducted with this view in mind.

The six experiments that follow examine the capacity of criterion bias to account for both frequency and semantic relatedness effects, by using nonword items that are in fact misspelled words (e.g., ENVLEOPE). These items, in which a pair of medial letters is transposed, can be readily perceived as words (Bruner \& O'Dowd, 1958) and produce strong interference effects in lexical decision tasks (Chambers, 1979), suggesting a high degree of similarity to real words. If criterion bias is acting, items that form misspellings of high-frequency words should be particularly prone to errors in a lexical decision task (word-nonword classification). When the misspelled version of a high-frequency word is presented, there is a relatively high probability that sufficient orthographic features will be available to provide the limited number needed to activate the corresponding detector. Thus, misspellings of high-frequency words such as VEGTEABLE and MEJDCINE should produce more false word recognitions than misspellings of lowfrequency words, such as BLUTSER or TRETSLE.

Theories of word recognition that rely exclusively on search sequence bias to explain frequency effects would not predict such an effect. Since all lexical entries are assumed to require the same amount of stimulus information for a successful match, the probability of a false recognition would be controlled solely by the similarity of the misspelled stimulus to an actual word. The first experiment was designed to test this prediction.

\section{EXPERIMENT 1}

In this experiment, subjects were presented high- and low-frequency words, incorrectly spelled high-frequency words, incorrectly spelled low-frequency words, and orthographically legal nonwords. The task was a lexical decision task, with the "yes" response reserved only for correctly spelled words. If criterion bias provides an adequate account of frequency effects, there should be more errors (i.e., responding "yes") on misspelled highfrequency words than on misspelled low-frequency words.

Since a major determinant of error rates was to be the perceived similarity to an actual word, it was important to make sure that this variable was controlled across the two frequency conditions. This is difficult to establish empirically, since ratings of similarity might in fact be influenced by frequency. That is, the stimulus VEGTEABLE might be rated as being similar to the word "vegetable" merely because the detector for "vegetable" is strongly activated by VEGTEABLE. To avoid this problem, we selected pairs of words that had very similar orthographic structure (e.g., "mother" and "bother") and showed the appropriate frequency differences. Misspelled versions were then prepared by transposing the same pair of letters in each word (e.g., MOHTER, BOHTER). 


\section{Method}

Subjects. Twenty Monash University undergraduate students volunteered to serve as subjects. All subjects were paid for their participation in the experiment.

Stimulus materials. There were five different conditions with 32 items in each. The conditions were high-frequency words (HFW), low-frequency words (LFW), high-frequency misspelled words (HFM), low-frequency misspelled words (LFM), and legal nonwords $(\mathrm{N})$. The word items were formed by selecting pairs of words that possessed marked orthographic similarity (one letter different) and also possessed substantial difference in frequency of usage according to the Kurera-Francis (1967) word count (KF). Thus the HFW items MOTHER (KF: 216), PRESENCE (KF: 76), MARKET (KF: 155) were paired with the LFW items BOTHER (KF: 22), PRETENCE (KF: 4), MARKER (KF: 5), The average frequency of the HFW items was KF: 132 and the average frequency of the LFW items was KF: 7. The misspelled word items were formed by transposing two of the letters within each word, excluding the beginning two letters and the end letter. Thus the HFM items MOHTER, PRESNECE, MAKRET were paired with the LFM items BOHTER, PRETNECE, MAKRER. The $\mathrm{N}$ items were formed so as to match the word items in length. Thus $\mathrm{N}$ items were NORDLE, PLASBONT, DREPPEL, and so on. All items used in the experiment are listed in the appendix.

Two lists of items were formed so that while each list contained equal numbers of the five item types, no list contained both the misspelled and the properly spelled version of the same word. Ten subjects were run on each list.

Procedure. Stimulus items were arranged in a different pseudorandom order for each subject and presented on a computer-controlled video display. Twelve practice items were included. The subject rested the index finger of his preferred hand on a blue button and the index of his nonpreferred hand on a red button. He was instructed to press the blue button if the stimulus item was a valid English word and the red button if it was not. Subjects were told to respond as quickly and as accurately as possible. Each item was presented approximately $400 \mathrm{msec}$ after the subject depressed a foot switch. The stimulus item remained on the screen for a maximum of $4 \mathrm{sec}$ or until the subject made his response. When the response was made, the display was terminated and a message appeared informing the subject of the accuracy of the response. If the response was correct, the reaction time was also displayed.

\section{Results}

In this and all subsequent experiments, the effects of isolated trials with exceptionally long or short latencies were minimized by establishing cutoff points 2 standard deviations away from the mean for each subject and setting the outlying values equal to the cutoff. Trials on which an error was made were omitted. Mean reaction times and percentage error rates for each of the conditions are given in Table 1.

The nonword and word results were analyzed separately. For the nonwords, a 2 by 3 analysis of variance design was used; the factors were groups (subject groups in the subject analysis, item groups in the item analysis) and item type (HFM, LFM, N). For words, a 2 by 2 design was used; the first factor was groups, and the second was item type (HFW, LFW).

The nonword errors revealed a significant main effect of item type $\left[\mathrm{min} \mathrm{F}^{\prime}(2,92)=8.47, \mathrm{p}<.01\right]$. Orthogonal planned comparisons (Keppel, 1973) showed that HFM
Table 1

Mean Lexical Decision Times (in Milliseconds) and Percentage Error Rates (E) for Words and Misspelled Words as a Function of Frequency (Experiment 1)

\begin{tabular}{ccccc}
\hline $\begin{array}{c}\text { Condi- } \\
\text { tion }\end{array}$ & Example & $\begin{array}{c}\text { Correct } \\
\text { Response }\end{array}$ & RT & E \\
\hline HFW & MOTHER & Yes & 573 & 2.2 \\
LFW & BOTHER & Yes & 644 & 12.4 \\
HFM & MOHTER & No & 696 & 21.4 \\
LFM & BOHTER & No & 678 & 11.0 \\
N & NORDLE & No & 634 & 4.4 \\
\hline
\end{tabular}

items produced more errors than did LFM items [ $\mathrm{min} \mathrm{F}^{\prime}$ $(1,95)=7.26, p<.01]$, and misspelled items in general produced more errors than did legal nonword items $\left[\min F^{\prime}(2,92)=10.75, p<.01\right]$. The word data revealed a significant main effect of item type $\left[\min F^{\prime}(1,45)=9.94\right.$, $p<.01]$, indicating that fewer errors were made to HFW items than to LFW items.

Turning to the reaction time data, the nonword analysis produced a significant main effect of item type [min $\left.\mathrm{F}^{\prime}(2,90)=3.34, \mathrm{p}<.05\right]$. Orthogonal comparisons showed that HFM and LFM items did not differ [min $\left.\mathrm{F}^{\prime}(1,92)<1\right]$, but misspelled items took longer to classify than did legal nonwords $\left[\min \mathrm{F}^{\prime}(2,89)=6.34\right.$, $\mathrm{p}<.01]$. The word data produced a significant main effect of item type $\left[\min \mathrm{F}^{\prime}(1,32)=7.63, p<.01\right]$, indicating the presence of the well-established effect of frequency on lexical decision time.

\section{Discussion}

The results clearly show that the difference in error rate between high-frequency and low-frequency misspelled words was as predicted: The higher the frequency of the misspelled word, the more likely it was to be identified as a word. This would seem a major confirmation of a direct prediction of the criterion bias approach. The discovery that misspelled words tended to produce more errors than did legal nonwords is also as might be expected, given misspelled words' greater similarity to words.

Considering the reaction time data, it may be noted that the slower classification of misspelled words com. pared with legal nonwords has been observed previously (Chambers, 1979; Coltheart, Davelaar, Jonasson, \& Besner, 1977). This would not be directly predicted by Morton's $(1969,1970)$ version of criterion bias, as the most usual interpretation is that all nonwords would be classified at the same time, that is, when a deadline is reached following the failure of any logogen to discharge. However, Coltheart et al. have proposed a modification to the logogen model to account for the effect. They suggested that the overall level of activation in the logogens could be monitored, with the deadline for responding being extended if an appreciable increase in activation suggested that firing might be imminent. Misspelled words would be capable of producing this initial increase 
in activation. It may be noted that the addition of such an active monitoring system would mean that the logogen model loses a major distinguishing characteristic, that of being a completely passive word recognition system.

As indicated earlier, models of word recognition that incorporate a search sequence bias rather than a criterion bias cannot readily explain the greater proportion of errors for high-frequency misspelled words. In a lexical search model, the only variable capable of influencing error probabilities would be the similarity of the test items to actual words. If this is held constant across the HFM and LFM conditions, then the error rates should also be constant, since the only way that frequency affects access is by altering the order in which entries are examined.

However, close examination of the overall pattern of results suggests a way in which a search model might explain the effects. Elsewhere, it has been proposed (e.g., Chambers, 1979; Forster \& Bednall, 1976; Taft \& Forster, 1975) that discovery of a match between the stimulus and a lexical entry does not necessarily terminate the search process. If the criterion for a match is sufficiently low (although constant for all types of words), then it is possible that certain nonwords would exceed the criterion for a match and also that some words would match the wrong entry. In order to prevent stimuli's being misidentified, a subsequent detailed check of the orthographic properties of the stimulus is carried out. If the stimulus does not match the entry exactly, the match is then rejected, and the search resumes. The interruption of the search that this produces is held responsible for the increased time taken to reject nonwords that are similar to words, as occurred in Experiment 1 . If we now postulate that the orthographic check is not always carried out, or is not always accurately carried out (e.g., when under time pressure), then we can also explain why misspelled words produce more errors than legal nonwords. Any tendency to accept inappropriate matches would produce this result.

So far, this theory says nothing about the difference in error rates between HFM and LFM items. To explain this effect, it is only necessary to point out that more errors are made on low-frequency words (i.e., correctly spelled) than on high-frequency words. It is apparent that for some reason, a low-frequency word does not access the correct entry with the same probability as a high-frequency word. This would mean that lowfrequency misspelled words will produce fewer misidentifications than will high-frequency misspelled words, since an error will not occur if the relevant entry is never accessed.

However, this argument neglects the question of why frequency should affect probability of an entry's being accessed. The simplest explanation would be that many of the low-frequency items were absent from the subject's vocabulary. This is unlikely to be the sole explanation, given the absence of any very low-frequency words, the fact that college students served as subjects, and the fact that subjects who were questioned following the experiment subsequently revealed familiarity with the words upon which they had made errors. Therefore, this explanation seems to have been ruled out. However, an alternative explanation is not immediately apparent. Search models propose an exhaustive search that terminates only when an appropriate match is located or when all entries have been searched (in the case of a nonword). To explain why more errors are made on lowfrequency words, this aspect of the model would have to be modified.

One obvious possibility is that the decision making system does not always require a search to completion before making a "no" decision. Instead, a deadline is set so that if no entry has been accessed when the deadline is reached, a "no" decision is made (cf. Frederiksen \& Kroll, 1976). Presumably, such a deadline could be specified in terms of either the duration or, as is proposed here, the extent of the search. Whichever system was operating, the shorter the deadline, the faster would be the average "no" decision, and the more incorrect "no" responses would be made. For any given deadline, of course, the probability of an incorrect "no" response must be higher for a low-frequency word than for a highfrequency word, since the search for a low-frequency word takes longer.

This search model is depicted in Figure 1. The search proceeds from the entries with the highest frequency at the top of the figure toward the lowest frequency entries at the bottom. The search continues until a matching entry is found or the deadline is reached. As stated earlier, the deadline is considered to be determined by the number of entries searched, not the time for which the search has continued. When a matching entry is found, the postaccess check is carried out. For this to be effective, it is assumed that all the letters in the stimulus have been evaluated prior to the commencement of search (or at least by the time of the postaccess check). If the check fails to detect a mismatch between the stimulus and the entry, the search is terminated and the item is classified as a word. If a mismatch is detected, the entry is rejected and the search continues.

For the lexical decision task, Cases 1, 2, 5, 7,8, and 9 produce correct responses, whereas Cases 3,4 , and 6 lead to incorrect responses. Cases 4 and 6 represent instances in which the postaccess check has failed to detect a mismatch, and the item is incorrectly classified as a word. The probability of postaccess check failure is independent of frequency. Note also that incomplete postaccess checking leads to errors through failure to examine adequately those characteristics of the stimulus that diverge from the entry. Consequently, checking failure may lead to misspellings' being classified as words, but it would not lead to words' being classified as nonwords.

Cases 3 and 8 are of particular interest. In Case 3, the target entry lies beyond the deadline (whereas in Case 2 it does not), and therefore the stimulus is incorrectly 


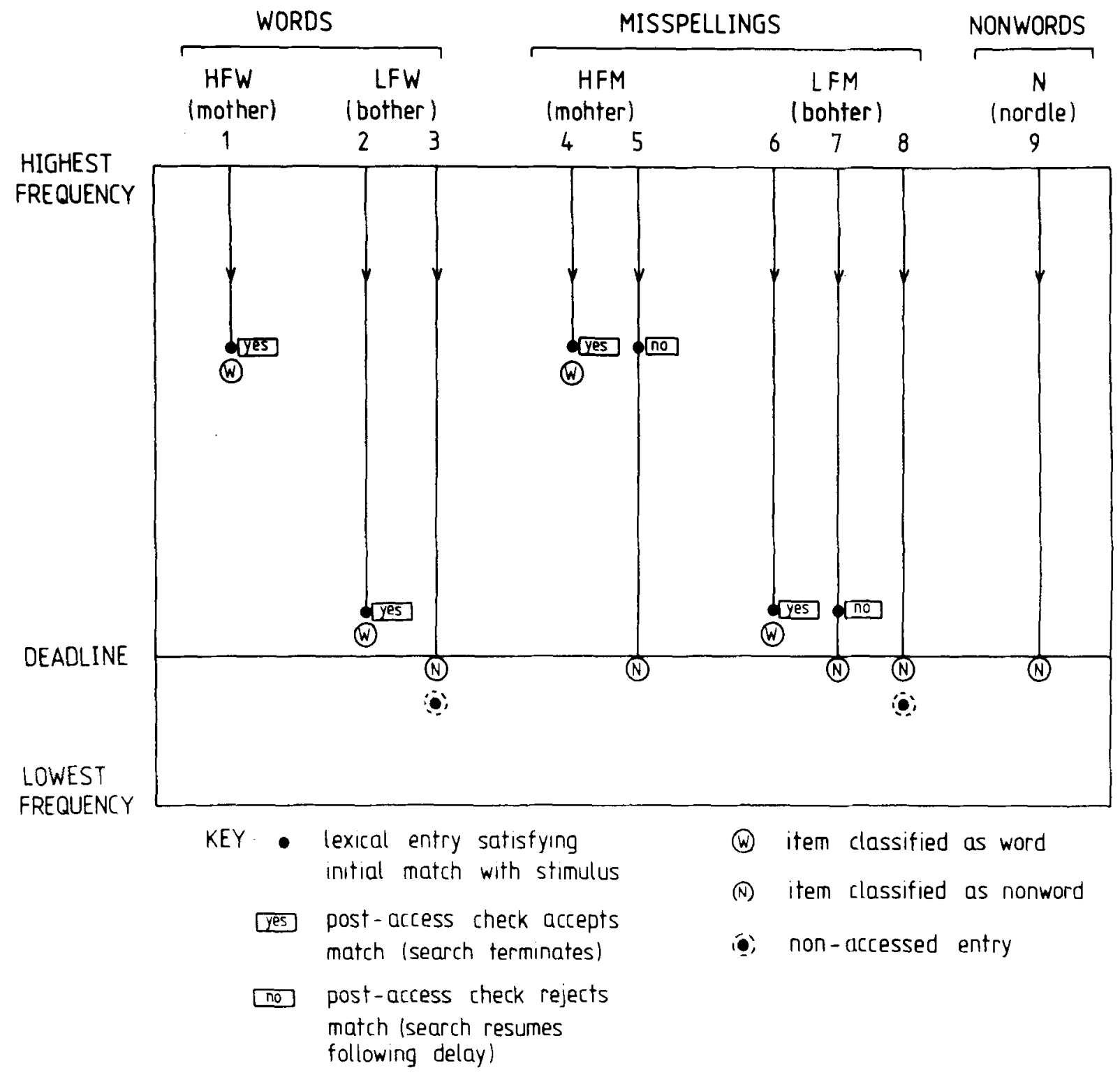

Figure 1. Schematic representation of deadline search model (for explanation, see text).

classified as a nonword. No such instance is listed for high-frequency items, on the assumption that the deadline would not be set so early in the search. Hence more errors will occur for LFW items than for HFW items. However, in Case 8, a matching entry lies beyond the deadline, but since the stimulus is an LFM item, failure to access the entry leads to the correct decision. Thus correct responses are obtained in two ways for the LFM items (Cases 7 and 8), whereas there is only one path to correct responses for the HFM items (Case 5). The added source of correct responses for LFM items (Case 8) means that LFM items exhibit fewer errors than do HFM items.

\section{EXPERIMENT 2}

So far, it has been shown that in a lexical decision task, low-frequency misspelled words produce fewer errors than do high-frequency misspelled words. This is what would be expected if criterion bias was responsible for word frequency effects, although it is possible to modify the search model in a way that might also account for such results. As mentioned earlier, the effects of semantic priming have also been interpreted as reflecting the action of a criterion bias mechanism. Meyer and Schvaneveldt (1976) have proposed a worddetector model in which semantic priming reflects the fact that the identification of a word leads to a decrease in the number of features that are required to be input to the word detectors of semantically related words before their thresholds are exceeded. If this is correct, then preceding a misspelled word by a semantically related word should act to increase the tendency to falsely identify the misspelling as a word.

This prediction was tested using a double lexical decision task (two items presented simultaneously), in 
which the same misspelled word was preceded either by a related word (e.g., DEER ANTLEOPE) or by an unrelated word (e.g., HULL ANTLEOPE).

\section{Method}

Subjects. Thirty Monash University undergraduates volunteered to serve as subjects. All were paid for their participation in the experiment.

Stimulus materials. A total of 288 two-word items were constructed; 128 of these consisted solely of words and 160 consisted of either a word and a misspelled word or a word and a legal nonword.

Items consisting of words only. Thirty-two pairs of semantically related words were constructed to serve as word/related word (W-RW) items (e.g., DEER ANTELOPE, GOLF CRICKET). These were matched with 32 word/unrelated word (W-W) items, prepared by removing the first word of each semantically related pair and replacing it with an unrelated word equivalent in length and Kuxera-Francis (1967) word frequency (e.g., HULL ANTELOPE, BONE CRICKET). An additional 64 pairs of unrelated words served as filler items.

Items consisting of a word and a misspelled word. Thirty-two word/related misspelled word (W-RM) items were formed by first constructing a pair of related words and then transposing two letters of the second word, excluding the first two letters and the last letter (e.g., LETTER ENVLEOPE, TOBACCO CIGRAETTE). These were matched with 32 word/unrelated misspelled word (W-M) items, formed by removing the word from each W-RM item and replacing it with an unrelated word equivalent in length and Kucera-Francis (1967) word frequency (e.g., POLICE ENVLEOPE, EMPEROR CIGRAETTE). A further 32 filler items were constructed in which the first item member was a misspelled word and the second was an unrelated word (e.g., WEIHGT CALM).

Items containing legal nonwords. Thirty-two word-nonword (W-N) items were formed by removing the misspelled words from the W-M items and replacing them with legal nonwords of equal length (e.g., POLICE MIRPREAT). Thirty-two filler items were also included in which a legal nonword preceded the word (e.g., PAMSLET CALM).

The items were formed into two lists so that no misspelled word, legal nonword, or word was found in more than one item in a list.

Procedure. The procedure was as in Experiment 1, except that two letter sequences appeared simultaneously on each trial (displayed left to right), and the subject was told to press the blue button if both members of an item were valid English words and to press the red button if at least one member was not.

\section{Results}

Mean reaction times and percentage error rates for each of the conditions are given in Table 2. As in Experiment 1 , items containing nonwords were analyzed separately from items exclusively composed of words.

Table 2

Mean Lexical Decision Times (in Milliseconds) and Percentage Error Rates (E) for Words and Misspelled Words as a Function of Semantic Relatedness of a Context Word (Experiment 2)

\begin{tabular}{llccr}
\hline $\begin{array}{c}\text { Condi- } \\
\text { tion }\end{array}$ & \multicolumn{1}{c}{ Example } & $\begin{array}{c}\text { Correct } \\
\text { Response }\end{array}$ & RT & \multicolumn{1}{c}{ E } \\
\hline W-RW & DEER ANTELOPE & Yes & 1254 & 6.0 \\
W-W & HULL ANTELOPE & Yes & 1312 & 11.7 \\
W-RM & LETTER ENVLEOPE & No & 1331 & 45.8 \\
W-M & POLICE ENVLEOPE & No & 1355 & 35.4 \\
W-N & POLICE MIRPREAT & No & 1260 & 13.1 \\
\hline
\end{tabular}

The nonword item errors revealed a significant main effect of item type $\left[\min F^{\prime}(2,114)=21.26, p<.01\right]$. Orthogonal planned comparisons revealed that W-RM items produced significantly more errors than did W-M items $\left[\min \mathrm{F}^{\prime}(1,115)=4.30, \mathrm{p}<.05\right]$ and $\mathrm{W}-\mathrm{RM}$ and W.M items together exhibited more errors than did W-N items $\left[\min F^{\prime}(2,114)=38.07, p<.01\right]$. Analysis of the error data for word items showed that significantly fewer errors were made to W-RW items than to W-W items $\left[\min F^{\prime}(1,57)=5.20, p<.05\right]$.

Turning to the reaction time data, the nonword item analysis produced a significant main effect of item type $\left[\min \mathrm{F}^{\prime}(2,114)=3.60, \mathrm{p}<.05\right]$. Orthogonal planned comparisons revealed that W-RM and W-M items did not significantly differ $\left[\min \mathrm{F}^{\prime}(1,78)<1\right]$ but that $\mathrm{W}$-RM and W-M items took longer to classify than did W-N items $\left[\min F^{\prime}(2,96)=11.97, p<.01\right]$, redemonstrating the interference effect discussed in Experiment 1. The word item data failed to reveal a significant difference between $W \cdot R W$ and $W-W$ items $\left[\min F^{\prime}(1,57)=3.07\right.$, $\mathrm{p}>.05]$, although this difference was significant in both subject and item analyses $\left[F_{1}(1,28)=7.22, p<.05\right.$; $\left.\mathrm{F}_{2}(1,36)=5.33, \mathrm{p}<.05\right]$.

\section{Discussion}

The results indicate clearly that the presence of a semantically related word leads to an increase in the false recognition rate of misspelled words, as might be expected if the effects of semantic priming are produced through criterion bias.

However, as in the previous experiment, this finding does not rule out alternative explanations. It has been proposed (e.g., Becker, 1979; Forster, 1976) that semantic priming results from the fact that it is possible to carry out two searches simultaneously. The first search is a frequency-ordered search through a set of entries defined on the basis of the orthographic properties of the target word. The second is a search through a set of entries that are semantically related to the context word (i.e., a semantically ordered search); if the stimulus is located faster in the semantic search than in the frequency search, then a semantic priming effect will occur. If two independent searches are in fact carried out, then related stimuli would have the opportunity to be matched with a lexical entry in either of two searches, and it would follow that semantically related misspelled words would consequently have increased opportunity to be falsely recognized. On the other hand, semantically unrelated misspellings would be limited to only one occasion upon which an inappropriate match could be made, namely, during the frequency-ordered search, and consequently would exhibit fewer false recognitions. As in Experiment 1, the basic notion being appealed to is that error incidence is determined by the probability of a stimulus's encountering its corresponding lexical entry. Although such an argument is somewhat ad hoc, it can. not be readily discarded.

So far, a prediction derived from the theory of crite. rion bias has been borne out with respect to both word 
frequency and lexical priming effects. However, in both cases, it has been possible to develop alternative explanations that do not involve a criterion bias assumption. A further attempt to demonstrate the presence of criterion bias entails a more direct examination of its proposed role in the rapid identification of words.

As already explained, the notion of criterion bias proposes that fast responses in a lexical decision task are the result of the premature acceptance of high-frequency or semantically related words, premature in the sense that some substantial proportion of the information in the stimulus has not been examined at the time the response is made. Thus, for example, Morton (1970) has proposed that "the advantage of high frequency words is that less evidence is required from a high frequency stimulus for a decision to be made" (p. 135) and "the presence of a context means that less information is required from a stimulus for that word to be recognised" (p. 115). For other items, low-frequency words in particular, identification occurs only when all (or nearly all) of the stimulus information has been processed. If this is so, it would follow that if classification was inhibited for every item until all stimulus information was processed, then no word would be identified faster than any other (if matched closely on orthography). In other words, the nature of criterion bias implies that if there was an item classification task that could be performed only by evaluating all stimulus information prior to responding, then, for this task, semantic priming and frequency effects would be suppressed.

One task that seems likely to possess the necessary properties is the misspelling decision task. In this task, the subject is asked whether or not an item is a misspelled version of a word. Items such as ENVLEOPE are positive instances, to which the subject responds "yes." All other items, including both correctly spelled words and nonwords, should be responded to with a "no" decision. As words and nonwords now share the same response, it is no longer reasonable to classify items on the basis of whether or not a stimulus accesses a lexical entry (unlike the lexical decision task); to satisfy the needs of the misspelling decision task, it is essential that subjects be able to specifically identify stimuli as misspelled words. To do this, it would seem necessary to access the words from which the misspellings were derived and then to carefully compare the lexical entry with the stimulus to determine whether the item is a misspelling or a correctly spelled word. For this to occur, the stimulus representation would need to be quite detailed, so as to allow each letter and its position to be clearly defined. Once a stimulus had been evaluated to the extent that letters and letter order could be clearly ascertained, it is unlikely that the amount of information left unprocessed would be sufficient to allow criterion bias (in any meaningful sense) to operate, given that a word is completely specified by its letters and their ordering.

This proposition may be illustrated in the case of frequency. Let $\mathrm{H}$ be the number of features (on average) that are required to activate high-frequency detectors, $L$ the number for low-frequency detectors, and $T$ the number of features needed to specify the letters and letter-order information accurately. Criterion bias models of the type we are considering here postulate that $\mathrm{H}<\mathrm{L} \leqslant \mathrm{T}$. Further assume that $\mathrm{p}$ is the rate at which stimulus features are extracted; this is assumed to be constant for all words. As the lexical decision times (LDT) for HFW and LFW items are determined by the time to activate the corresponding detector plus the time to make the appropriate decision (d), it follows that $\mathrm{LDT}(\mathrm{HFW})=\mathrm{Hp}+\mathrm{d}$ and $\mathrm{LDT}(\mathrm{LFW})=\mathrm{Lp}+\mathrm{d}$; since $\mathrm{H}<\mathrm{L}$, LDT(HFW) $<\mathrm{LDT}(\mathrm{LFW})$. However, in a misspelling decision task, decisions cannot be reached when the appropriate detector is activated. Instead, it is necessary to wait until sufficient features are available to carry out an accurate postaccess check, that is, until $\mathbf{T}$ features have been extracted ( $T$ need not, of course, be the total set of features available in the stimulus). Therefore, misspelling decision times (MDT) are as follows, where $c$ is the time to carry out the orthographic check: $\operatorname{MDT}(\mathrm{HFW})=\mathrm{Tp}+\mathrm{c}+\mathrm{d}$ and $\mathrm{MDT}(\mathrm{LFW})=\mathrm{Tp}+\mathrm{c}+\mathrm{d}$; that is, MDT(HFW) $=$ MDT(LFW).

It needs to be emphasized that in misspelling decision, the detectors relevant to high-frequency misspelled words would still be activated faster than the detectors for lowfrequency misspelled words. However, this would not lead to faster "yes" responses, since it would still be necessary to wait until $\mathrm{T}$ features are available so that a detailed examination of the features of the stimulus could be carried out to determine whether it is a correctly spelled word or a misspelling.

The foregoing analysis holds only if it is assumed that a detailed orthographic check is possible after the detector has been activated. In an extreme version of logogen theory that emphasized the passive nature of word recognition, it might be proposed that such a check could not be carried out, and therefore, responses would be controlled solely by whether a logogen was fired. Such a model would predict normal frequency effects for words in a misspelling decision task, but it would also predict an extremely high error rate (near 100\%) for misspelled words. If the misspelled word activates a logogen, it will be classified as a word (incorrect), and if it fails to activate a logogen, then it will be classified as a nonword (also incorrect).

If, on the other hand, a less passive approach is adopted, and a postactivation orthographic check is allowed, then correct responses for high-frequency misspelled words should be no faster than those for lowfrequency misspelled words. The same prediction applies to responses to correctly spelled words, since, once again, $\mathrm{T}$ features must be extracted before the stimulus item can be correctly classified.

Much the same line of reasoning applies to the semantic priming effect. If $R$ is the number of features required to activate the detectors of primed words and $U$ is the number required for unprimed words, then the criterion 
bias approach postulates that $\mathrm{R}<\mathrm{U} \leqslant \mathrm{T}$. For the lexical decision task, faster responses to related pairs is due to the fact that $R<U$. This also explains the higher error rate for W-RM items. However, for the misspelling decision task, $T$ features must be extracted, and hence semantic relatedness should be unrelated to response times for both misspelled words and correctly spelled words. The next two experiments parallel the first two, except that the misspelling decision task is used rather than the lexical decision task.

\section{EXPERIMENT 3}

This experiment was designed to establish whether or not subjects are able to classify stimuli accurately as misspelled words and, assuming this to be the case, whether frequency still influences reaction times and error rates. The expectation from a criterion bias model is that the normal effect of frequency on RT would be absent in this task (or at least markedly reduced) both for correctly and for incorrectly spelled words. This stems from the fact that a correct word/misspelled word discrimination cannot be made before the stimulus features have been carefully checked, and hence criterion bias mechanisms would be no longer free to operate.

The misspelling decision task was used, in which subjects were required to view a string of letters and decide as quickly as possible whether a letter string represented a misspelling of a valid English word. The distractors were composed of correctly spelled words and legal nonwords.

\section{Method}

Subjects. Twenty Monash University undergraduates volunteered to serve as subjects. All subjects were paid for their participation in the experiment.

Stimulus materials. The items were the same as those used in Experiment 1.

Procedure. The procedure was the same as that in Experiment 1 , with the exception that subjects were now to press the blue button if the stimulus was a misspelling of a valid English word. They were to press the red button if it was not, that is, if it was a properly spelled word or a legal nonword.

Examples of each of the three item types were shown to the subjects, and the subjects were questioned to ensure that they understood the task. They were told that all misspelled words would be identical with normal words except that a pair of letters had been reversed. It was emphasized that the blue button was to be pressed as soon as it was determined that an item could represent a misspelling of an English word.

\section{Results}

Mean reaction times and percentage error rates for each condition are given in Table 3.

The results for words and misspelled words were analyzed in a 2 by 2 by 2 design; the factors were groups, item type (misspelled/correctly spelled), and frequency (high or low). Considering first the reaction time data, there was a significant main effect of frequency [min $F^{\prime}$ $(1,64)=16.65, p<.01]$, indicating that high-frequency items were classified faster than were low-frequency items. There was also a significant main effect of item
Table 3

Mean Misspelling Decision Times (in Milliseconds) and Percentage Error Rates (E) as a Function of Frequency (Experiment 3)

\begin{tabular}{ccccc}
\hline $\begin{array}{c}\text { Condi- } \\
\text { tion }\end{array}$ & Example & $\begin{array}{c}\text { Correct } \\
\text { Response }\end{array}$ & RT & E \\
\hline HFW & MOTHER & No & 1020 & 4.7 \\
LFW & BOTHER & No & 1157 & 9.4 \\
HFM & MOHTER & Yes & 1258 & 14.6 \\
LFM & BOHTER & Yes & 1454 & 25.9 \\
N & NORDLE & No & 1481 & 21.6 \\
\hline
\end{tabular}

type (misspelled/correctly spelled) $\left[\min F^{\prime}(1,43)=24.08\right.$, $\mathrm{p}<.01]$, with misspelled words ("yes" response) taking longer to classify than words ("no" response). The interaction between item type and frequency was not significant $\left[\min F^{\prime}(1,75)<1\right]$. An examination of the simple main effect (Keppel, 1973) confirmed that HFM items were classified significantly faster than were LFM items $\left[\min F^{\prime}(1,65)=11.30, \quad p<.01\right]$. Further analysis revealed that legal nonwords were classified significantly more slowly than were HFM items $\left[\min F^{\prime}(1,38)=7.56\right.$, $\mathrm{p}<.01]$, but legal nonwords did not differ from LFM items $\left[\mathrm{min} \mathrm{F}^{\prime}(1,47)<1\right]$.

Turning to the error data, both the main effects of frequency and item type were significant $\left[\mathrm{min} \mathrm{F}^{\prime}\right.$ $(1,66)=6.77, p<.01$, and $\min \mathrm{F}^{\prime}(1,30)=14.54, \mathrm{p}<.01$, respectively]. The interaction between frequency and item type was not significant $\left[\min \mathrm{F}^{\prime}(1,64)=1.18\right.$, $p>.05]$. It is interesting to note that there were now fewer errors made to HFM items than to LFM items, the reverse effect to that found in Experiment 1 [examination of the simple main effect confirmed this: $\left.\min F^{\prime}(1,64)=7.06, p<.01\right]$. Legal nonwords did not differ from either HFM or LFM items [min $F^{\prime}$ $\left.(1,33)=1.15, p>.05 ; \min F^{\prime}(1,47)<1\right]$.

\section{Discussion}

The most important feature of the results is that a strong frequency effect is maintained in the misspelling decision task, both for correctly and for incorrectly spelled words and there is no evidence of a reduction in the size of the effect compared with the results obtained in a lexical decision task. This result strongly opposes a criterion bias explanation of frequency effects.

Furthermore, the error rates for high-frequency words and misspellings are lower than those for low-frequency words and misspellings. This would not be expected if for high-frequency items, the discrimination between word and misspelling were made on the basis of relatively inferior stimulus information.

A logogen model incorporating criterion bias would need to be modified if it were to encompass these results. First of all, it would have to explain why misspelled words appear to be so much more capable of accessing lexical entries when the misspelling decision task is being performed than when the lexical decision task is being performed; this is indicated by the fact that the error 
rates for misspelled words in the lexical decision task are much lower than the correct classification rates in the misspelling decision task. Second, it would have to explain how the discrimination between words and misspellings is made; some active checking mechanism would need to be introduced to explain this. Finally, it would need to explain why this ability to successfully discriminate does not result in the elimination or reduction of the frequency effect.

While the misspelling decision results are inconsistent with the operation of criterion bias, it appears that a search sequence bias model could explain the obtained results. Postaccess orthographic checking is a commonly postulated operation and would be required in both the lexical decision and in the misspelling decision tasks. Since frequency influences only the order in which entries are searched, there is no reason to expect frequency effects to be modified in the misspelling decision task. Provided that the misspelled item is sufficiently similar to a real word to guarantee that the search will stop at the appropriate entry, the entry for a highfrequency word will be accessed faster than that for a low-frequency word whether the stimulus is correctly spelled or not. Once access has been made, the check could be readily carried out, since in the search model considered here, all feature extraction is assumed to have been completed before the search begins.

The question may be raised as to why the frequency effect for misspelled words obtained in this experiment was not obtained in Experiment 1, in which the lexical decision task was used. This result can be explained by a search model. In the present task, when the postaccess orthographic check reveals that the item is in fact a misspelling, a "yes" decision can be made immediately, since part of the task is to detect misspellings. But in the lexical decision task, a decision cannot be made at this point, since there may be some other entry that matches the stimulus exactly (for example, the stimulus SLAT cannot be classified as a nonword merely because it is a misspelling of "salt"). Hence, before a "no" decision for misspelled words can be made, the search must be continued until it is discovered that no other entry matches the stimulus. Since this search will be exhaustive (or will continue until some deadline is reached), no frequency effects would be expected. This result parallels the results obtained by Forster and Bednall (1976) with a syntactic function task. This task required subjects to decide whether words of varying frequencies belonged to a specific syntactic category, such as noun or verb. When the target category was "noun," highfrequency nouns such as WIFE produced faster "yes" responses than did low-frequency nouns such as COBRA. But when the target category was "verb," the same sets of nouns failed to produce frequency effects. This reflects the fact that WIFE cannot be classified as nonverb merely on the basis of the first entry discovered, as examples such as "suit" and "iron" readily demonstrate.

A possible objection to this argument could be made by pointing out that the same reasoning should be applied to the misspelling decision task. Subjects should not classify a word item until an exhaustive search has established that it is not a misspelling of some other word. That is, SALT could not be classified as a word when the entry for the word "salt" is discovered, since the stimulus SALT is also a misspelling of "slat." This reasoning leads to the prediction that frequency effects for word items should not be found, which of course is incorrect. However, in this task, subjects were specifically instructed to respond as soon as the item could be classified into any one of the three categories word, misspelled word, or nonword. Hence there is no requirement to extend the search beyond the initial discovery of a matching entry. That is, for all items except nonwords, a terminating search is sufficient, and hence frequency effects are to be expected.

One aspect of the data that is not immediately explained by a search model is the slower classification of misspelled words than of normal words. Indeed, one would expect that a "yes" response for high-frequency misspelled words would be faster than a "no" decision for low-frequency words (other things being equal), given the order in which lexical entries are presumed to be encountered. Advocates of the search approach would need to propose that this peculiarity reflects the activity of some postaccess process, possibly during the matching phase. Perhaps the rejection of a highly similar but different match is more "difficult" than the acceptance of an identical match.

Finally, some attention needs to be directed to the error data. The reversal of the error difference between high-frequency and low-frequency misspelled words is a striking result, and one that might be expected if the version of the lexical search model outline in Experiment 1 is accepted. To recapitulate, in Experiment 1 , it was proposed that high-frequency misspellings were more likely to access lexical entries than were lowfrequency misspellings. This was attributed to the operation of a deadline mechanism, in which the search terminates before it locates some low-frequency entries. In a lexical decision task (Experiment 1), this failure to access a low-frequency entry enhances performance on misspelled words, since it is the discovery of a closely matching entry that produces errors. Thus low-frequency misspelled words produce a lower error rate than do high-frequency misspelled words. However, in a misspelling decision task, exactly the reverse is the case. Failure to access an entry means that a misspelled word will be erroneously classified as a nonword, and hence low-frequency misspelled words will produce a higher error rate than will high-frequency misspelled words. The conclusion to be drawn from this experiment, then, is that frequency effects can be demonstrated in a task in which the role that criterion bias mechanisms could play has been reduced to an absolute minimum. It now remains to see whether the same can be said of semantic priming effects. 


\section{EXPERIMENT 4}

This experiment examines whether the time taken to recognize items such as DOTCOR as misspelled versions of real words can be decreased by providing a correctly spelled context word such as NURSE. The expectation derived from criterion bias explanations of semantic priming would be that no such effects would be present.

\section{Method}

The task was the misspelling decision task, as in the previous experiment, except that an item pair was presented, and subjects were asked to check both members for misspelling. A "yes" response meant that one or both members were misspelled words, and a "no" response signified that neither was a misspelled word. The items and design were the same as those used in Experiment 2, except for the delection of $32 \mathrm{~W}$-W filler items. A total of 30 volunteer undergraduates served as subjects.

\section{Results}

Mean reaction times and percent error rates for each of the conditions are given in Table 4 .

The data for the W-RW, W-W, W-RM, and W-M conditions were analyzed in a 2 by 2 by 2 design; the factors were groups, context (related-unrelated), and item type (misspelled word/word). Considering first the reaction time data, the main effect of context was significant $\left[\min \mathrm{F}^{\prime}(1,80)=6.99, \mathrm{p}<.01\right]$, whereas the main effect of item type (misspelling/word) did not approach significance $\left(\min \mathrm{F}^{\prime}<1\right)$. There was no evidence of an interaction between context and item type $\left[\min F^{\prime}(1,87)=.17, p>.05\right]$, and the presence of a priming effect for misspelled words alone was confirmed on examination of the simple main effect $\left[\min \mathrm{F}^{\prime}(1,80)=4.49, \mathrm{p}<.05\right]$.

Turning to the error data, the main effect of context was not significant $\left[\min \mathrm{F}^{\prime}(1,73)<1\right]$, but there was a significant effect of item type $\left[\min \mathrm{F}^{\prime}(1,64)=16.38\right.$, $\mathrm{p}<.01 \mathrm{~J}$; misspelled words produced more errors than did words. The interaction between context and item type was not significant $\left[\min F^{\prime}(1,67)=1.88, p>.05\right]$.

The W-N items were classified more slowly than were W-RM items $\left[\min F^{\prime}(1,55)=6.64, p<.05\right]$, but they did not differ significantly from W-M items $\left[\min \mathrm{F}^{\prime}(1,54)=1.88, \mathrm{p}>.05\right]$. W-N items exhibited fewer errors than did W-RM items $\left[\min F^{\prime}(1,54)=4.19\right.$, $\mathrm{p}<.05]$ and did not differ from W-M items $\left[\min \mathrm{F}^{\prime}(1,53)\right.$ $=2.07, \mathrm{p}>.05]$.

Table 4

Mean Misspelling Decision Times (in Milliseconds) and Percentage Error Rates (E) as a Function of Context (Experiment 4)

\begin{tabular}{lllcc}
\hline $\begin{array}{c}\text { Condi- } \\
\text { tion }\end{array}$ & \multicolumn{1}{c}{ Example } & $\begin{array}{c}\text { Correct } \\
\text { Response }\end{array}$ & RT & E \\
\hline W-RW & DEER ANTELOPE & No & 1768 & 4.6 \\
W-W & HULL ANTELOPE & No & 1839 & 6.4 \\
W-RM & LETTER ENVLEOPE & Yes & 1773 & 20.4 \\
W-M & POLICE ENVLEOPE & Yes & 1876 & 16.4 \\
W-N & POLICE MIRPREAT & No & 1974 & 11.3 \\
\hline
\end{tabular}

\section{Discussion}

The results offer little support for criterion bias explanations of the semantic priming effect. To discriminate misspelled words from words and legal nonwords, a detailed examination of the orthography of the stimulus is necessary. This did not prevent semantic relatedness from teducing the latency of the classification process. As with word frequency, the search model would seem to offer a more appropriate way of viewing priming effects.

To comment on the error data, it is necessary to recall the lexical search position described in Experiment 2 . In the lexical decision task, misspelled words that had been preceded by related words were seen to produce more errors because of the opportunity to form inappropriate lexical matches in either of two searches. If an accessed entry was appropriately rejected in the semantically based search, it could still be inappropriately accepted in the frequency-ordered search. In the misspelling decision task, related misspelled words would again have two opportunities to access lexical entries, and this might be expected to now lead to more successful identification. Of this, there was no evidence, and so this result to some extent opposes the lexical search explanation. However, it should be pointed out that this effect would only be expected if a similar effect occurs for correctly spelled words, and it is clear in Table 4 that this was certainly not marked (whereas inspection of the results in Table 2 for the lexical decision task show a more marked error difference for correctly spelled words).

So far, the argument against the criterion bias approach has rested on the crucial assumption that an item cannot be identified as a misspelled word until all (or nearly all) of the orthographic information in the stimulus has been processed. Roughly, the argument is equivalent to saying that although the appropriate word detector may be activated by a subset of letters in the word, there is no way of deciding whether the stimulus is a word or a misspelled word until all the letters have been identified, and it is this that prevents criterion bias from operating. There is, however, an alternative proposal that might circumvent this difficulty. Suppose that after the appropriate detector has exceeded threshold, the subsequent checking operation immediately makes use of the raw physical stimulus, instead of awaiting a more fully encoded orthographic representation. If a perfect physical match occurs, the stimulus is identified as a word, and a "no" response is made. If a discrepancy is detected, the stimulus is classified as a misspelled word, and a "yes" response is made. If no detector at all is activated, the item is designated as a nonword, and a "no" response follows. Since the detectors for high-frequency words will be activated faster than for low-frequency words, no matter whether the stimulus is correctly spelled or not, it follows that frequency effects should still be observed in the misspelling decision task. 
Becker (1979) and Becker and Killion (1977) have proposed a word recognition system that appears to have these properties. They suggest that a visual stimulus is initially processed by a feature analyzer whose action results in a number of word detectors exceeding threshold. To determine which of the detectors is appropriate to the stimulus, a "verification stage" is proposed in which a detector is used to construct a representation of the word that can be compared with the raw stimulus in memory.

The major weakness in this kind of theory is the problem of specifying how a detector could be used to construct a representation of the stimulus in raw physical terms. The detector could activate only some abstract orthographic representation of the word (such as its spelling), and it would be quite absurd to propose that all possible physical realizations of the word could be made available. To avoid this problem, one would have to postulate an intermediate translation device that would convert the orthographic representation into the appropriate format. For example, if the preceding context consists entirely of uppercase letters of a certain font, then the translator would use this information to construct an appropriate uppercase representation. This proposal runs into problems in the case of nonuniform character sets, such as handwriting, in which the same letter is seldom realized twice in the same way. However, since word recognition experiments typically use standard fonts, it could be claimed that this problem can be overcome, even if only for standard uniform fonts.

These considerations suggest a way in which this view of the verification process could be tested. If the stimulus is presented in randomly mixed case (e.g., mOthEr), then there is no way in which the precise visual form of the stimulus could be predicted. A mismatch in the verification stage could be due simply to an unexpected alteration of case. The next two experiments were designed to determine whether the frequency and semantic priming effects were in any way affected by this procedure.

\section{EXPERIMENTS 5 AND 6}

\section{Experiment 5}

The method, design, and items of this experiment were exactly the same as for Experiment 3 , except that a new group of 20 subjects was used and the letters were altered from uppercase to lowercase at random with a probability of .5. As in Experiment 3, the misspelling decision task was used. The results are shown in Table 5.

Comparison of Tables 3 and 5 shows that, except for a general lengthening of reaction times, almost exactly the same pattern of results is found in the two experiments. The effect of frequency on reaction time in Table 5 was significant $\left[\min \mathrm{F}^{\prime}(1,76)=8.28, \mathrm{p}<.01\right]$, as was the effect of item type (misspelled/word) $\left[\min \mathrm{F}^{\prime}(1,59)=18.90, \mathrm{p}<.01\right]$, but there was no interaction between these effects $\left[\min \mathrm{F}^{\prime}(1,61)=1.14\right.$, $p>.05]$. This pattern was repeated for the error data.
Table 5

Mean Misspelling Decision Times (in Milliseconds) for Mixed-Case Items as a Function of Frequency (Experiment 5)

\begin{tabular}{cllcr}
\hline $\begin{array}{c}\text { Condi- } \\
\text { tion }\end{array}$ & Example & $\begin{array}{c}\text { Correct } \\
\text { Response }\end{array}$ & RT & E \\
\hline HFW & mOthEr & No & 1223 & 4.1 \\
LFW & Bother & No & 1315 & 7.2 \\
HFM & moHTer & Yes & 1438 & 19.1 \\
LFM & BohtER & Yes & 1610 & 35.3 \\
N & NOrDLE & No & 1654 & 8.7 \\
\hline
\end{tabular}

Table 6

Mean Misspelling Decision Times (in Milliseconds) for Mixed-Case Items as a Function of Context (Experiment 6)

\begin{tabular}{llccr}
\hline $\begin{array}{c}\text { Condi- } \\
\text { tion }\end{array}$ & \multicolumn{1}{c}{ Example } & $\begin{array}{c}\text { Correct } \\
\text { Response }\end{array}$ & RT & E \\
\hline W-RW & deER anTELope & No & 2104 & 7.8 \\
W-W & hULL aNTeLOpe & No & 2181 & 10.0 \\
W-RM & Letter eNvLEoPe & Yes & 1990 & 22.3 \\
W-M & POLICE eNvLEoPe & Yes & 2121 & 26.9 \\
W-N & PoLICe MirprEAT & No & 2242 & 12.8 \\
\hline
\end{tabular}

The magnitudes of the frequency effects shown in Table 5 ( $92 \mathrm{msec}$ for words and $172 \mathrm{msec}$ for misspelled words) are slightly less than those shown in Table 3 (137 msec and $196 \mathrm{msec}$ ). However, combining the results of both experiments into a single design indicated that this reduction was not significant, since there were no significant interactions between frequency and case (uppercase/mixed case) ( $\min \mathrm{F}^{\prime}<1$ in each instance). This analysis did, however (not surprisingly), reveal a significant effect of frequency [for words, $\left.\min F^{\prime}(1,64)=11.50, p<.01\right]$; for misspellings, $\min F^{\prime}$ $(1,52)=12.92, \mathrm{p}<.01]$, whereas case type was not significant [for words, $\min \mathrm{F}^{\prime}(1,46)=3.78, \mathrm{p}>.05$; for misspellings, min $\left.F^{\prime}(1,42)=2.53, p>.05\right]$.

\section{Experiment 6}

The results of the previous experiment show clearly that the frequency effects observed in the misspelling decision task are quite unaffected by random scrambling of case. The present experiment checks whether the same conclusion applies to the semantic priming effect. The method, design, materials, and procedure were the same as for Experiment 4, except that, once again, a new sample of 30 subjects was used and the case of each letter was randomly varied.

The results are shown in Table 6. Once again a related context word has a significant effect on misspelling decision times $\left[\min \mathrm{F}^{\prime}(1,88)=7.58, \mathrm{p}<.01\right]$, and this effect did not interact with item type (misspelled/word) (min $\left.F^{\prime}<1\right)$. The priming effects ( $77 \mathrm{msec}$ for words and $131 \mathrm{msec}$ for misspelled words) were slightly (although not significantly) larger than those obtained for uppercase items in Experiment 4 ( $71 \mathrm{msec}$ and $103 \mathrm{msec}$ ), hence there is no support for 
the notion that scrambled case might attenuate the effects. This was further demonstrated upon combining the reaction time data from Experiments 4 and 6 , in which there was found to be a significant effect of priming [words, min $F^{\prime}(1,78)=5.92, p<.05$; misspellings, $\left.\min F^{\prime}(1,63)=8.25, p<.01\right]$ and of case [words $\min \mathrm{F}^{\prime}(1,62)=9.65, \quad \mathrm{p}<.01$; misspellings, $\left.\min F^{\prime}(1,67)=4.55, \quad p<.05\right]$, although there was no interaction between priming and case [words, $\min \mathrm{F}^{\prime}(1,78)=.01 ;$ misspellings, $\left.\min \mathrm{F}^{\prime}(1,86)=.23\right]$.

The error data showed the same pattern found in Experiment 4 . There were significantly more errors for misspelled words than for words $\left[\min F^{\prime}(1,85)=16.59\right.$, $p<.01]$, but there was no effect of context on errors $\left[\min F^{\prime}(1,85)=2.10, p>.05\right]$, nor was there any interaction between these effects $\left(\min F^{\prime}<1\right)$.

\section{Discussion of Experiments 5 and 6}

The results of Experiments 5 and 6 show that the effects of frequency and semantic relatedness are unaffected by the scrambled-case manipulation. The verification model would expect that this would severely impair item classification, given the great difficulty in generating visual representations of the stimuli (e.g., a seven-letter word would have $2^{7}$ possible case orders). Disregarding the verification model, the data are inconsistent with criterion bias models generally. The tendency for increased classification time with scrambled-case stimuli indicates that word recognition is slowed by this unusual structuring. For the logogen model, this would suggest a slowing in the rate at which appropriate features are accumulated by target logogens, which in turn (as mentioned in the introduction) should result in larger priming and frequency effects (cf. Becker \& Killion, 1977). However, there is little evidence of this; to take the example of semantic priming, scrambling case led to a significant increase in classification time, but there was no evidence of a significant increase in priming.

For a search model, there is no such problem. Any tendency for a delay in classification time due to scram. bled case would reflect an increase in the time needed to form the standard letter code necessary for comparison with lexical entries. As this occurs presearch, no interaction would be expected with frequency or priming effects.

\section{GENERAL DISCUSSION}

The aim of the preceding six experiments was to evaluate the proposal that both frequency effects and semantic priming effects could be adequately explained in terms of a criterion bias mechanism. The essence of such a mechanism is the notion that some words are recognized faster than others because they require less information to be extracted from the stimulus.

The first two experiments provide strong support for this mechanism. These experiments showed that nonwords are much more likely to be misidentified as words if they are similar to high-frequency words rather than to low-frequency words. In addition, such errors are also more likely if the previous lexical context is semantically related to the misspelling. These findings are to be expected if responses in a word recognition experiment are typically made before all information in the stimulus has been analyzed and if the amount of information needed is influences by frequency or context.

The corollary of this view, namely, that these findings should not be obtained if premature responding is prevented, was tested in the remaining four experiments. These experiments required the subject to specifically detect misspelled words, and hence one can be sure that a correct "yes" response to an item such as ENVLEOPE means that a subject is aware of the fact that it is similar to but different from ENVELOPE. Similarly, a correct "no" response to ENVELOPE means that the subject is aware of the fact that it is identical to a real word. It is difficult to see how this task could be performed without waiting until virtually all orthographic information had been processed (Antos, 1979, reports a similar approach to investigating criterion bias, although employing only the lexical decision task).

These experiments proved to be very damaging to the criterion bias model. Experiments 3 and 4 demonstrated that correct misspelling decision times still showed both frequency and semantic priming effects. Faced with this evidence, the only way to preserve criterion bias is to argue that premature responses still occur in the misspelling decision task, but in such a way as to avoid absurdly high error rates. One possible procedure for achieving this is the verification model of Becker and Killion (1977), in which postaccess orthographic checking is carried out on the basis of some global comparison of the physical stimulus with the expected form derived from the activated detector; thus spelling errors might be detected even though the relevant segments of the stimulus had not yet been analyzed. However, for this to work, the physical shape of the uninterpreted segments would have to be perfectly predictable. With completely regular stimuli, such as words printed entirely in uppercase characters of the same font, this may be possible, but with words printed in randomly varied case, this seems highly unlikely. Yet random variation of case had no effect on either the frequency effect or the semantic priming effect, as shown by the final two misspelling decision experiments. Hence this particular line of escape seems to be ruled out.

There is one further proposal that might be made in the interest of preserving the criterion bias approach, and that is to adopt a modified view of the postaccess check. Suppose that when the detector is activated, enough features have been extracted from the stimulus to determine whether the stimulus contains the correct letter sequence, regardless of case. For example, suppose that the detector for "mother" is activated when only the letters MO--ER have been identified. Although there is insufficient information extracted to enable the medial letters to be identified by themselves, there 
might be sufficient information available to decide whether these letters could be either TH (in which case the item is classified as a word) or HT (in which case it is classified as a misspelled word). That is, sufficient features are available for a postaccess orthographic check to be carried out, but not for the letters to be identified independently.

This view of the postaccess check will allow the system to respond correctly in the misspelling decision task and yet still show frequency and priming effects. However, it is important to note that this benefit is not obtained without cost, for there is doubt as to whether this model should really be thought of as a criterion bias model.

To see why this is the case, recall the earlier discussion of the criterion bias approach to the frequency effect. It was argued that the criterion bias model proposes that $\mathrm{H}<\mathrm{L} \leqslant \mathrm{T}$, where $\mathrm{H}$ is the number of features required to activate a high-frequency detector, $L$ is the number required for a low-frequency detector, and $T$ is the number required for accurate postaccess checking. Since the above modification to the criterion bias model assumes that when any detector fires, there is sufficient information to carry out an accurate check, it follows that $\mathrm{T}=\mathrm{H}<\mathrm{L}$. The major difficulty we see with this proposal is that it fails to explain why $\mathrm{H}<\mathrm{L}$. Why must $\mathrm{L}$ features be extracted for a low-frequency word when $\mathrm{H}$ features would be enough for accurate identification? Why not set the thresholds for lowfrequency word detectors at the same level as those for high-frequency detectors? Similar problems arise in the treatment of semantic priming, for which the modified theory would propose that $T=R<U$. Why should the number of features required for unrelated pairs $(U)$ be greater than the number required for accurate identification $(T)$ ? Resolution of this problem requires further theoretical development, which will not be attempted here. For the present purposes, it will suffice merely to point out that the evidence presented here is relevant only to criterion bias models proposing that $\mathrm{H}<\mathrm{L} \leqslant \mathrm{T}$ or $\mathrm{R}<\mathrm{U} \leqslant \mathrm{T}$. We believe that such models are ruled out by the results obtained with the misspelling decision task.

Perhaps it should be pointed out that the evidence presented here cannot be taken as a general disconfirma. tion of the logogen model. There is no reason to suppose that this theory is necessarily committed to criterion bias assumptions of the sort we have discussed here. There are several alternatives that could be explored. For example, it could be proposed that logogens vary in their sensitivity to the presence of relevant features. High-frequency logogens have the same thresholds as low-frequency logogens, but the growth of activation in the high-frequency logogen takes place at a faster rate. With the additional assumptions of postaccess checking and a deadline, this theory could explain many of the results reported here in much the same way as the search model does.
There are no doubt more complicated ways of extending the logogen model. However, the advantages of pursuing such modifications are lessened by the fact that an alternative type of bias, namely, search sequence bias, appears to be able to explain the observed effects reasonably well. The results obtained with the misspelling decision task offer no problems at all for theories using this type of bias. Correct responses for both words and misspelled words require the location of a closely matching entry, followed by an orthographic check to determine whether or not the stimulus item is correctly spelled. Since both frequency and semantic context influence the order in which entries are searched, it follows that these variables should still influence response times whether the task is lexical decision or misspelling decision.

Obviously, the major concern of the lexical search model is the results of Experiments 1 and 2. The distributions of errors observed in the lexical decision task (Experiments 1 and 2) are compatible with a search sequence bias model, but only in a post hoc fashion. To explain the effect of frequency on the misidentification of misspelled words, it was necessary to postulate a lower probability of access for low-frequency words, this being a result of adopting too brief a search. To explain why a related semantic context word increases the number of misidentifications, it was necessary to appeal to the idea that there are two different searches capable of locating the relevant entry, and hence there is twice the probability of discovering the matching entry (and making an error). In fact, the error results scarcely qualify as successful predictions from a search sequence bias model, although they do count as successful predictions from a criterion bias model. The status of search sequence bias would be improved substantially if there were further predictions that could be derived. One such prediction concerns the effect of frequency on errors. Using suitable instructions (e.g., emphasizing accuracy), it should be possible to increase the length of the deadline adopted by subjects. With longer searches, there should be no difference in error rates for correctly spelled high- and low-frequency words. If this can be achieved, then it should also be the case that there are no differences in error rates for incorrectly spelled highand low-frequency words. If this is not the case, then the deadline explanation becomes less reasonable. Of course, if this could be achieved only at the expense of losing the frequency effect on reaction time, then a criterion bias explanation would still be tenable.

One final aspect of the results that deserves mention is the fact that both the frequency and semantic priming effects tend to be greater in the misspelling decision task than in the lexical decision task, as is the overall response time. This is clearly not in agreement with a lexical search theory, which would predict equal effects in the two tasks. One possible explanation is that the search process itself is slower in the misspelling decision task. Subjects generally reported this task to be quite 
difficult, largely due to the fact that considerable care was required not to misclassify misspelled words as nonwords. This may have led to a tradeoff between search speed and the probability of accessing an entry with imperfect stimuli. It seems reasonable to assume that search rate is critically dependent on the time required to make the initial comparison between each entry and the internal representation of the stimulus (as distinct from the postaccess check). If this comparison has to take into account possible misspellings, then it may well take longer.

It is interesting to compare the results reported here with those previously reported using degraded stimuli. It could be thought that diminishing the visual quality of stimuli might also lead to a slower search rate. This theory would make the correct prediction in the case of the semantic priming effect, since this effect is indeed increased for degraded stimuli (Becker \& Killion, 1977; Meyer et al., 1975). However, it would not make the correct prediction in the case of the frequency effect, since this has been shown to be independent of visual quality (Becker \& Killion, 1977; Stanners et al., 1975). The explanation for this apparent conflict in results may be that the effects of degradation were restricted to the encoding stage. In both the Becker and Killion (1977) and the Stanners et al. (1975) studies, the stimuli were quite legible; they were not of such poor visual quality as to prevent the extraction of the essential features. Unlike misspelled words, these items would have been capable of providing the unambiguous information necessary to ensure lexical access. A slightly extended encoding stage could have allowed the construction of an effective stimulus representation, and as this would contain no deliberately misleading information, the search process could occur normally and the frequency effect would remain unchanged. To then explain the interaction between degradation and semantic priming, it need only be proposed that the tendency to rely on contextual information increases as stimulus quality decreases (cf. Forster, 1976). This seems a not unreasonable expectation, and there is ample evidence that semantic priming may be influenced by strategic factors (e.g., Neely, 1977; Tweedy, Lapinski, \& Schvaneveldt, 1977). In terms of search theory, this would mean increasing the priority of a semantically ordered search, a process very different from that of decreasing the rate of search.

There are other features of the results that are still unclear; for example, why are misspelled words identified much more slowly than normal words in frequency experiments, but not so in semantic priming experiments? The search model is not immediately able to explain this difference. It is conceivable that the effect (if reliable) occurs at the decision level, although no reason for this appears obvious. Further investigation may require a reformulation of the processes of frequencyordered vs. semantically ordered search.

\section{REFERENCES}

Antos, S. J. Processing facilitation in a lexical decision task. Journal of Experimental Psychology: Human Perception and Performance, 1979, 5, 527-545.

Becker, C. A. Semantic context and word frequency effects in visual word recognition. Journal of Experimental Psychology: Human Perception and Performance, 1979, 5, 252-259.

Becker, C. A., \& Killion, T. H. Interaction of visual and cognitive effects in word recognition. Journal of Experimental Psychology: Human Perception and Performance, 1977, 3, 389-401

Bednall, E. S. Frequency, semantic priming and ambiguity effects in word recognition. Unpublished doctoral thesis, Monash University, 1978

Broadbent, D. E. Word-frequency effect and response bias. Psychological Review, 1967, 74, 1-15.

Bruner, J. S., \& O'Dowd, D. A note on the informativeness of parts of words. Language and Speech, 1958, 1, 98-101.

Chambers, $\mathrm{S}$. M. Letter and order information in lexical access. Journal of Verbal Learning and Verbal Behavior, 1979, 18 225-241

Coitheart, M., Davelaar, E., Jonasson, J., \& Besner, D. Access to the internal lexicon. In S. Dornic (Ed.), Attention and performance VI. Hillsdale, N.J: Erlbaum, 1977.

Forsten, K. I. Accessing the mental lexicon. In R. J. Wales \& E. C. T. Walker (Eds.), New approaches to language mechanisms. Amsterdam: North Holland, 1976.

Forster, K. I., \& Bednale, E. S. Terminating and exhaustive search in lexical access. Memory \& Cognition, 1976, 4, 53-61.

Frederiksen, J. R., \& Kroll, J. F. Spelling and sound: Approaches to the internal lexicon. Journal of Experimental Psychology: Human Perception and Performance, 1976, 2, 361. 379.

KePpEL, G. Design and analysis: A researcher's handbook. Englewood Cliffs, N.J: Prentice-Hall, 1973.

Kućera, H., \& Francis, W. N. Computational analysis of present-day American English. Providence, R.I: Brown University Press, 1967.

Meyer, D. E., \& Schvaneveldt, R. W. Meaning, memory structure, and mental processes. Science, 1976, 192, 27-33.

Meyer, D. E., Schvanfyeldt, R. W., \& Ruddy, M. G. Loci of contextual effects on visual word recognition. In P. M. A. Rabbitt \& S. Dornic (Eds.), Attention and performance $V$. New York: Academic Press, 1975.

Morton, J. Interaction of information in word recognition. Psychological Review, 1969, 76, 165-178.

Morton, J. A functional model for memory. In D. A. Norman (Ed.), Model of human memory. New York: Academic Press, 1970

NEELY, J. H. Semantic priming and retrieval from lexical memory: Roles of inhibitionless spreading activation and limited capacity attention. Journal of Experimental Psychology: General, 1977, 106, 226-259.

Rubenstein, H., Garfield, L., \& Millikan, J. A. Homographic entries in the internal lexicon. Journal of Verbal Learning and Verbal Behavior, 1970, 9, 487.494.

Stanners, R. F., \& Forbach, G. B. Analysis of letter string. in word recognition. Journal of Experimental Psychologv, 1973, 98, 31-35.

Stanners, R. F., Jasthzkmbski, J. E., \& Westbrook, A Frequency and visual quality in a word-nonword classification task. Journal of Verhal Learning and Verbal Behavior, 1975. 14, 259-264.

TAFT, M., \& Fonstik, K. I. Lexical storage and retrieval of prefixed words. Journal of Verhal Learning and Verbal Behavior, $1975,14,638-647$.

Twefid, J. R., Lapinski. R. H., \& Schuanfeveidt, R. W Semantic context effects in word recognition: Influence of 
varying the proportion of items presented in an appropriate context. Memory \& Cognition, 1977, 5, 84-89.

\section{APPENDIX}

Items Used in Experiments 1, 3, and 5

\section{High-Frequency Words}

List 1. MOTHER, CULTURE, PROPER, IMPACT, MARKET, BROTHER, WORKING, DESPITE, SWITCH, LETTER, DINNER, PRESENCE, SOUND, NUMBER, RAISED, STRIKE.

List 2. REGION, NOTICE, PERSON, ATTACK, SUPPLY, PISTOL, EXPECT, MIDDLE, SUMMER, FOLLOW, MIXTURE, AROUND, SINGLE, WAITING, CATTLE, TRAINING.

\section{Low-Frequency Words}

List 1. LEGION, NOVICE, PARSON, ATTACH, SUPPLE, PISTON, EXPERT, MUDDLE, SIMMER, HOLLOW, FIXTURE, ABOUND, MINGLE, WAILING, CASTLE, TRAILING.

List 2. BOTHER, VULTURE, PROPEL, IMPART, MARKER, BROTHEL, WORDING, DESPISE, TWITCH, LITTER, SINNER, PRETENCE, HOUND, LUMBER, RAIDED, STRIFE.

High-Frequency Misspelled Words

List 1. TRIANING, CATLTE, WAITNIG, SINLGE, ARUOND, MITXURE, FOLOLW, SUMEMR, MIDLDE, EXPCET, PITSOL, SUPLPY, ATATCK, PESRON, NOTCIE, REIGON.

List 2. STIRKE, RIASED, NUBMER, SONUD, PRESNECE, DINENR, LETETR, SIWTCH, DEPSITE, WOKRING, BROHTER, MAKRET, IMPCAT, PROEPR, CUTLURE, MOHTER.

\section{Low-Frequency Misspelled Words}

List 1. STIRFE, RIADED, LUBMER, HONUD, PRETNECE, SINENR, LITETR, TIWTCH, DEPSISE, WODRING, BROHTEL, MAKRER, IMPRAT, PROEPL, VUTLURE, BOHTER.

List 2. TRIALING, CATSLE, WAILNIG, MINLGE, ABUOND, FITXURE, HOLOLW, SIMEMR, MUDLDE, EXPRET, PITSON, SUPLPE, ATATCH, PASRON, NOVCIE, LEIGON.

\section{Nonwords}

List 1. CAYPTE, FAIPTE, NOLDLE, BLANT, PLASBONT, DREPPEL, DALPEC, FLITTLE, KALPERT, SPLEATCH, PROMNET, STAPON, GAFDIN, PLINSH, MONRENT, LAPSIEST.

List 2. LURSTRON, NORDLE, BREABES, STIMCH, MARPLE, DAIVING, LEDDIE, STELPY, PHUPLE, CULSIG, MATLED, FLAPEY, PLORST, THIDLE, GLURST, PRENTE.

\section{Items Used in Experiments 2, 4, and 6}

\section{Word/Related Misspelled Word Pairs}

List 1. LETTER ENVLEOPE, SPIDER INSCET, NEEDLE THRAED, RELIGION CHRUCH, CHEESE BISCIUTS, HUNGRY THRISTY, HAND FIGNER, BED BLAKNET, STREET AVNEUE, SQUARE CICRLE, QUESTION ANWSER, DRAMA THAETRE, DEVICE GAGDET, DOCTOR MEIDCINE, TOWN CONUTRY, DARK NIHGT

List 2. TOBACCO CIGRAETTE, BUTTER MARAGRINE, JOY HAPPNIESS, LOTION LIUQID, HEAD SHOUDLERS, DOOR WIDNOW, HOUSE GADREN, NURSE HOSIPTAL,
SOLDIER SALIOR, LAW JUTSICE, RIVER STRAEM, THIEF BURLGAR, CABBAGE VEGTEABLE, CIRCUS ELEHPANT, GOD HEVAEN, RUG CAPRET.

\section{Word/Misspelled Word Pairs}

List 1. EMPEROR CIGRAETTE, MIRROR MARAGRINE, RAW HAPPNIESS, BALLAD LIUQID, CITY SHOUDLERS, LINE WIDNOW, SCHOOL GADREN, STEAM HOSIPTAL, PALACE SALIOR, CAR JUTSICE, HEART STRAEM, NIECE BURLGAR, RADIATOR VEGTEABLE, CANOE ELEHPANT, ACT HEVAEN, NUT CAPRET

List 2. POLICE ENVLEOPE, PILLAR INSCET, PARENT THRAED, CHARACTER CHRUCH, HAMMER BISCIUTS, NERVOUS THRISTY, ROOM FIGNER, NOTE BLAKNET, MONEY AVNEUE, MARKET CICRLE, POSITION ANWSER, CLOTH THAETRE, VISION GAGDET, BRIDGE MEIDCINE, GIRL CONUTRY, EAST NIHGT.

\section{Word-Nonword Pairs}

List 1. POLICE MIRPREAT, PILLAR GALPENG, PARENT SHERPESS, CHARACTER FLARST, HAMMER ARCLEMPT, NERVOUS CRENLT, ROOM MATEST, NOTE BLURSTE, MONEY FASPER, MARKET RANTEP, POSITION FATTLE, CLOTH EXSLOAT, VISION SEASART, BRIDGE TOSTLERT, GIRL SHILPER, EAST TRIDLE.

List 2. EMPEROR MIRPREAT, MIRROR GALPENG, RAW SHERPESS, BALLAD FLARST, CITY ARCLEMPT, LINE CRENLT, SCHOOL MATEST, STEAM BLURSTE, PALACE FASPER, CAR RANTEP, HEART FATTLE, NIECE EXSLOAT, RADIATOR SEASART, CANOE TOSTLERT, ACT SHILPER, NUT TRIDLE.

\section{Word/Related Word Pairs}

List 1. TEACHER STUDENT, DAGGER KNIFE, LOST FOUND, DEER ANTELOPE, PRIZE TROPHY, MOTHER DAUGHTER, WINTER AUTUMN, NEGATIVE POSITIVE, WEDDING FUNERAL, LUXURY EXTRAVAGANCE, PRIMARY SECONDARY, MAXIMUM MINIMUM, COURAGE COWARDICE, DANGER SAFETY, HUGE MASSIVE, REMOTE ISOLATED.

List 2. THUNDER LIGHTNING, TUNA SALMON, JEWELLER DIAMOND, COMPLEX DIFFICULT, OSTRICH FEATHER, POETRY MUSIC, SMOOTH ROUGH, COPY DUPLICATE, GOLF CRICKET, ANGER HATRED, WORLD UNIVERSE, BACON SAUSAGE, PLUMBER MECHANIC, LOVE EMOTION, OCEAN MARINE, INSPECT SURVEY.

\section{Word-Word Pairs}

List 1. CURTAIN LIGHTNING, MALT SALMON, SERPENT DIAMOND, PROJECT DIFFICULT, LEISURE FEATHER, BATTLE MUSIC, MENTAL ROUGH, PLOT DUPLICATE, BONE CRICKET, TIRED HATRED, WATER UNIVERSE, ELBOW SAUSAGE, PILGRIM MECHANIC, COST EMOTION, SUGAR MARINE, RECOVER SURVEY.

List 2. CHAPTER STUDENT, EMBRYO KNIFE, COLD FOUND, HULL ANTELOPE, CLOUD TROPHY, FUTURE DAUGHTER, REGION AUTUMN, ADVANCED POSITIVE, PARTNER FUNERAL, PLANET EXTRAVAGANCE, POPULAR SECONDARY, REALITY MINIMUM, GESTURE COWARDICE, ANIMAL SAFETY, SEAT MASSIVE, DEBATE ISOLATED.

(Received for publication March 7, 1980; revision accepted September 3, 1980.) 